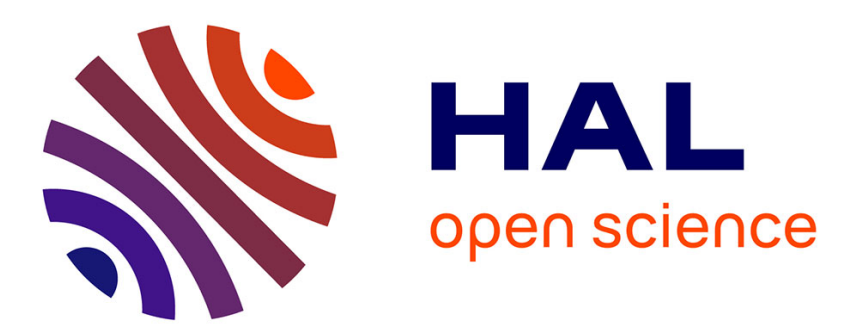

\title{
Towards embedded detection of polyps in WCE images for early diagnosis of colorectal cancer
}

\author{
Juan S. Silva, Aymeric Histace, Olivier Romain, Xavier Dray, Bertrand
}

Granado

\section{- To cite this version:}

Juan S. Silva, Aymeric Histace, Olivier Romain, Xavier Dray, Bertrand Granado. Towards embedded detection of polyps in WCE images for early diagnosis of colorectal cancer. International Journal of Computer Assisted Radiology and Surgery, 2014, 9 (2), pp.283-293. 10.1007/s11548-013-0926-3 . hal-00843459

\section{HAL Id: hal-00843459 \\ https://hal.science/hal-00843459}

Submitted on 7 Dec 2014

HAL is a multi-disciplinary open access archive for the deposit and dissemination of scientific research documents, whether they are published or not. The documents may come from teaching and research institutions in France or abroad, or from public or private research centers.
L'archive ouverte pluridisciplinaire HAL, est destinée au dépôt et à la diffusion de documents scientifiques de niveau recherche, publiés ou non, émanant des établissements d'enseignement et de recherche français ou étrangers, des laboratoires publics ou privés. 


\title{
Towards embedded detection of polyps in WCE images for early diagnosis of colorectal cancer
}

\author{
Juan Silva · Aymeric Histace • Olivier \\ Romain • Xavier Dray • Bertrand Granado •
}

Received: date / Accepted: date

\begin{abstract}
Purpose: Wireless capsule endoscopy (WCE) is commonly used for noninvasive gastrointestinal tract evaluation, including the detection of mucosal polyps. A new embeddable method for polyp detection in wireless capsule endoscopic images was developed and tested.

Methods: First, possible polyps within the image were extracted using geometric shape features. Next, the candidate regions of interest were evaluated with a boosting-based method using textural features. Each step was carefully chosen to accommodate hardware implementation constraints. The method's performance was evaluated on WCE datasets including 300 images with polyps and 1200 images without polyps. Hardware implementation of the proposed approach was evaluated to quantitatively demonstrate the feasibility of such integration into the WCE itself.

Results: The boosting-based polyp classification demonstrated a sensitivity of $91.0 \%$, a specificity of $95.2 \%$ and a false detection rate of $4.8 \%$. This performance is close to that reported recently in systems developed for an on-line analysis of video colonoscopy images.

Conclusion: A new method for polyp detection in videoendoscopic WCE examinations was developed using boosting-based approach. This method achieved good classification performance and can be implemented in situ with embedded hardware.

Keywords Colorectal cancer · polyp · WCE · videoendoscopy · boosting · co-occurence matrix $\cdot$ Hardware implementation

J. Silva, A. Histace and O. Romain

ETIS UMR CNRS 8051, ENSEA-University of Cergy-Pontoise, 6 av. du Ponceau 95000, France Tel.: $+33(1) 34256834$

Fax: $+33(1) 34256920$

E-mail: \{aymeric.histace, olivier.romain\}@u-cergy.fr

X. Dray

iTEC/CRB3 lab, Paris Diderot, Paris 7 University, France

B. Granado

LIP6 Lab, University Pierre et Marie Curie, CNRS, Paris, France
\end{abstract}




\section{Introduction}

\section{Colorectal Cancer}

Colorectal cancer (CRC) is the first cause of death by cancer in developed countries, with an estimated incidence of 728.550 cases worldwide in 2008, with fatal outcome in $43 \%$ of cases. Overall, CRC is the third more frequent cancer after lung cancer and breast cancer [1]. Prevention of CRC by detection and removal of preneoplastic lesions (colorectal adenomas) is therefore of paramount importance and has become a worldwide public health priority. Currently, colonoscopy is the the "gold standard" technique for diagnosis of colorectal adenoma and cancer. Using a videoendoscope, gastroenterologists can perform and record a complete examination of the colon in order to detect and to remove suspicious tissular structures like adenomas which degenerescence could lead to cancer. Because colonoscopy is performed under general anesthesia, mini-invasive techniques such as computed-tomography-based colonography and wireless capsule endoscopy (WCE) have been developed. Both techniques are currently considered valid alternative options to videocolonoscopy in patients with contra-indication or low compliance to general anesthesia. WCE takes form of a pill equipped with a CCD or CMOS sensor, two batteries, and a RF (radiofrequency) transmitter, that enables the wireless identification of gastrointestinal abnormalities such as ulcers, blood and polyps [2] with no need for hospitalization or sedation. In the last decade, WCE has become a breakthrough technology for diagnosis of small bowel pathologies [3]. Many fabricants such as Given Imaging, IntroMedic, and Olympus [4] have developed a variety of capsules for the complete examination of the gastrointestinal tract.

Practically speaking, after ingestion of the capsule, about 50,000 images are captured along the digestive tract and each of them are wirelessly transmitted to a wearable receiver and saved for a postponed physician's reading. Off-line image processing enables the identification of gastrointestinal abnormalities (like the aforementioned polyps and adenoma) by the gastroenterologist.

Current main issues of WCE are:

- The complete analysis of the 50,000+ images is time-consuming for physicians, and even for experienced ones, WCE diagnoses are sometimes challenging.

- The transmission of the 50,000+ images, that represents $80 \%$ of the overall energy consumption of the embedded batteries, limits to 8 hours the autonomy of the classic WCE, whereas 12 hours are necessary to scan the complete intestinal tract.

- A recent study comparing diagnostic capabilities of videoendoscopy and of WCE shows that the average detection rate is around $80 \%$ polyps per patient [3,5]. Thus, the improvement of polyp detection and classification capabilities of WCE is expected from gastroenterologists.

- Processing capabilities of WCE are limited to transmit raw images. No "intelligence" is currently embedded into the imaging device itself.

In the context of early diagnosis of colorectal adenoma and cancer, the "Cyclope" project proposes a new generation of WCE [6] (see figure 3 for illustration) that will 
permit an in situ detection of the polyps and, consequently, to only emit the images which are important for the final diagnosis. The expected benefits are twofold:

1. An increase of the battery lifetime up to 12 hours considering the fact that, except for particular pathologies, only a small percentage of the 50,000 images would contain polyps and will be consequently transmitted (see figure 1).
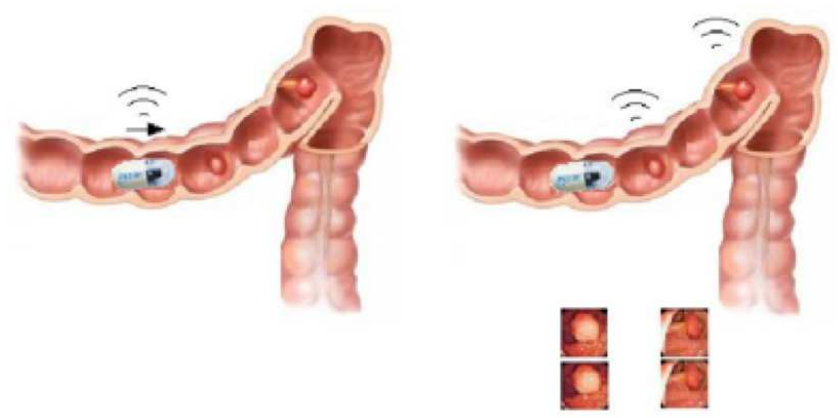

Fig. 1 Comparison between continuous transmission (left) and intelligent one (right).

2. A facilitated off-line final diagnosis for the clinician considering the law amount of transmitted data after in situ hardware processing and the possibility to highlight particular regions of interest within the images that possibly contain a polyp.

In figure 2, a comparison between the usual clinical workflow and the expected one with Cyclope WCE is proposed.

In [6] and [7], a first prototype demonstrator equipped with an active stereo vision sensor was proposed to detect protuberating polyps within the colon. The proposed embedded detection algorithm used a SVM classifier trained on robust 3D feature descriptors. The overall detection performance was very promising with a global classification rate of $97 \%$ on an in vitro dataset consisting of 111 polyps (40 adenomas and 81 hyperplasias) made in silicon. Nevertheless, it appears that for real case examinations, $3 \mathrm{D}$ features are not sufficient to detect the large variety of polyp shapes that can be very flat at an early evolution stage.

In this article, we focus on the $2 \mathrm{D}$ analysis of the videoendoscopy images in order to investigate other possibilities than 3D shape characterization of polyps to improve capabilities of WCE. As in [7], a particular attention is given to propose a global detection/classification scheme that can be integrated into the "Cyclope"-WCE architecture shown in figure 3 and more precisely, by taking benefits of the FPGA block.

The remainder of this article is organized as follows: a state-of-the-art on detection of polyps in videocolonoscopy using $2 \mathrm{D}$ features is proposed in Section 2. In Section 3 , the proposed approach is detailed. Experimental results are given in Section 4. Discussion, with a particular focus on hardware implementation, and conclusion are given in the last two sections. 


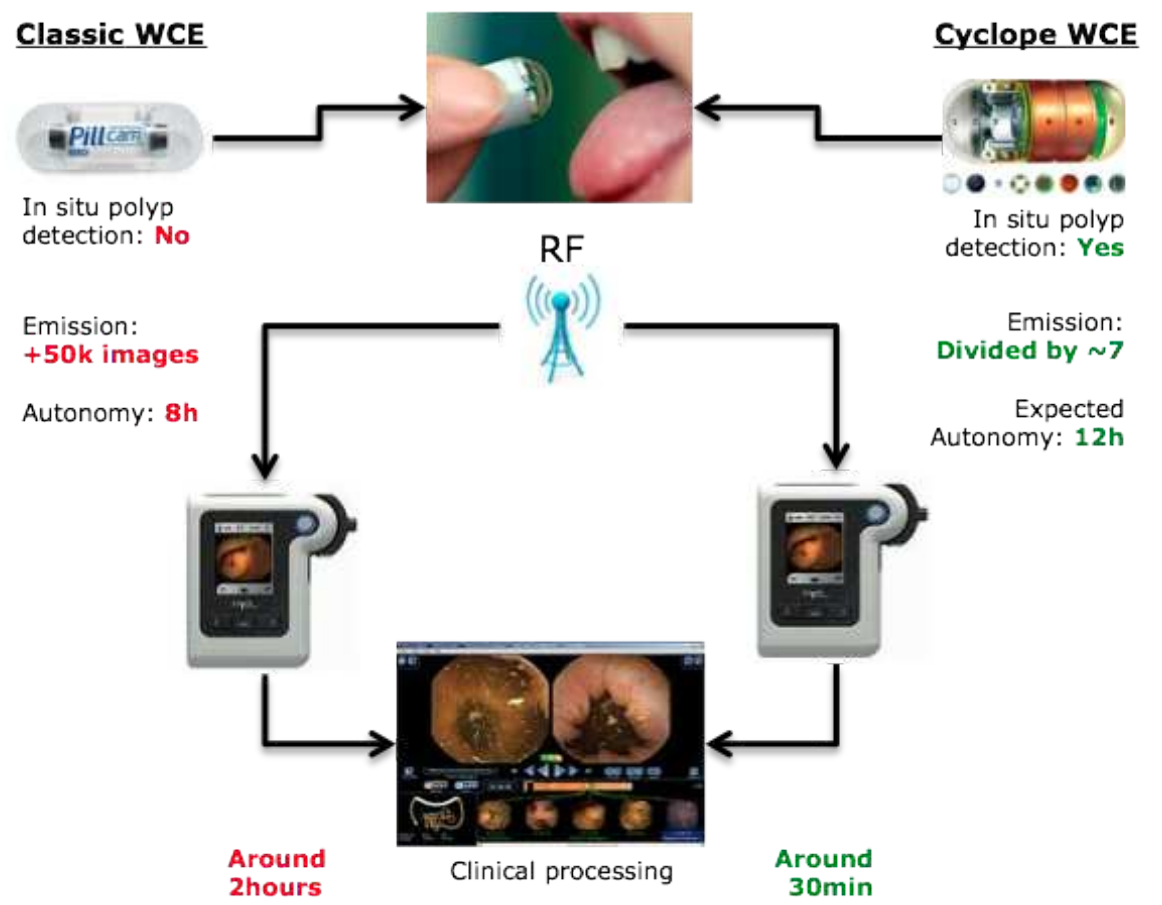

Fig. 2 Comparison of the classic clinical workflow (left) and the expected one using Cyclope WCE (right), with corresponding improvement in green.

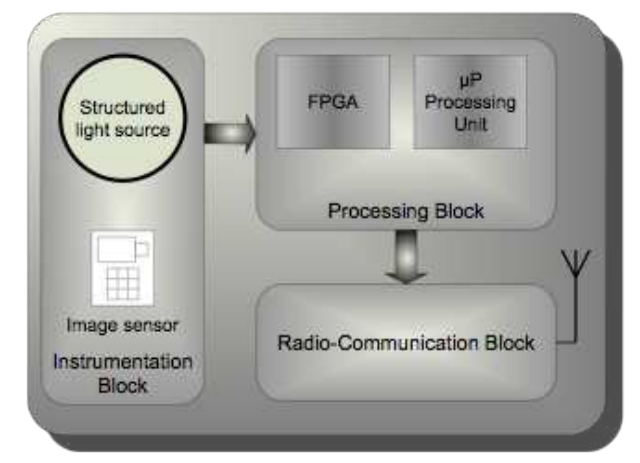

Fig. 3 Block Diagram of the "Cyclope WCE"

\section{Related Works}

Several previous references have considered the detection of intestinal polyps in videocolonoscopy images in the last few years ([8-12] among recent ones). They are mainly divided into two categories: those based on geometric features of the polyps (size and shape) and those based on textural features.

In the framework of "Cyclope project", we focused our attention on four particular recent contributions. 
In [9], Bernal et al. authors propose a study made on videoendoscopy images. They developed a region descriptor based on the depth of valleys (SA-DOVA). Resulting algorithm, divided into several steps, including region segmentation, region description and region classification, is characterized by promising detection performance (see Tab. 1).

In [10], Figueiredo et al. assume that polyps show up as protrusions that can be detected using the local curvature of the image. Consequently, a method based on the mean and geometric curvature of the WCE image is proposed. The main drawback of the proposed approach is the strong dependance on the protrusion measure of the polyp to identify potential candidates. The consequence is that if a polyp is not protruding enough from the surrounding mucosal folds, it may be missed.

In [11], Karargyris and Bourbakis propose an algorithm for WCE images mainly based on Log Gabor filters and Susan edge detector. Based on the geometric information of the resulting detected ROI, a level-set segmentation is then initialized for an accurate delineation of the polyps. On the considered WCE image database (10 polyps and 40 non-polyps), the method gives satisfying results but authors highlight that the taking into account of texture or color-based features within the detection/classification scheme would significantly increase related performance.

Finally, Kodogioannis and Boulougoura [12] propose a texture-based approach. Authors introduce new texture-based features computed from the chromatic and achromatic spectra of the Region of Interest (ROI) that may contain a polyp. For classification, a neurofuzzy scheme is proposed. Main result is that the textural information is of first importance for the discrimination between polyps and non-polyps.

Table 1 summarizes the main principle and the obtained performance of these four main contributions.

\begin{tabular}{|c|l|l||l|}
\hline Authors & $\begin{array}{l}\text { Main princi- } \\
\text { ple }\end{array}$ & $\begin{array}{l}\text { Classification per- } \\
\text { formance }\end{array}$ & Database \\
\hline Fernal [9] & Geometry & $\begin{array}{l}\text { Sensitivity } 89 \% \text { Speci- } \\
\text { ficity } 98 \%\end{array}$ & $\begin{array}{l}300 \text { videocolonoscopy im- } \\
\text { ages containing a polyp } \\
\text { (freely available) }\end{array}$ \\
\hline Karkargyris [1] & Geometry & $\begin{array}{l}\text { No indicated perfor- } \\
\text { mance } \\
\text { ficity } 67.5 \%\end{array}$ & $\begin{array}{l}17 \text { WCE videos of 100 im- } \\
\text { ages each, containing ex- } \\
\text { ample of polyps (10), flat } \\
\text { lesions, diverticula, bub- } \\
\text { bles, and trash liquids }\end{array}$ \\
\hline Kodogiannis [12] & Texture & $\begin{array}{l}\text { Sensitivity 97\% Speci- } \\
\text { ficity 94\% }\end{array}$ & $\begin{array}{l}50 \text { WCE images (10 polyps } \\
\text { and 40 non-polyps) }\end{array}$ \\
\hline
\end{tabular}

Table 1 Main characteristics of 4 of the most recent references of the literature.

All four presented approaches for polyp detection and classification are definitely of primary interest but may not fully compel to the hardware constraints of Cyclope architecture (the detection algorithm is to be embedded in the FPGA block of limited resources) since all developed methods were designed mainly for an off-line use by the clinician and can fully benefit from the high computing capabilities of the lastgeneration processors: As a consequence, the related processing schemes include possible demanding algorithms like active contour segmentation [11], blob detector [9] or 
local curvature estimation [10], that have not been proved yet to be easily embedded on a "low" resource hardware like FPGA. Moreover, it also appears that image databases used for performance estimation are size-limited and/or not freely available for possible comparison, except in the case of [9], more particularly when considering WCE images.

Taking benefits of the aforementioned reference, and taking into account the heavy hardware constraints of "Cyclope" WCE, we propose in this article a learning-based polyp detection approach using texture-based descriptors. In order to compare related performance to the most recent literature, we will use for illustration the database freely provided by $[9]$.

\section{Methods}

The proposed method is inspired from the psychovisual methodology used by the physician when doing an endoscopic examination: First, a detection of the Regions of Interests (ROI) that may contain a polyp is performed using shape and size features extracted from the image. This first pre-selection allows a first fast scanning of the image. Once the ROI are detected, a second analysis, based on texture (homogeneity, granularity, coarseness...) is achieved. Practically speaking, we propose a global scheme for the detection/classification of possible polyps divided into two steps:

1. Considering the geometric step of the proposed approach, simple image processing tools make possible the detection of circular/elliptical shape like the Hough transform for instance.

2. The texture-based classification is the main keypoint of the global scheme since the rejection of most of the false positive preselected ROI have to be performed at this stage. To achieve this, we propose to design an ad hoc classifier based on a boosting-based learning process using textural features.

The global scheme of this approach is summarized in figure 4. Each step is detailed in the following sections.

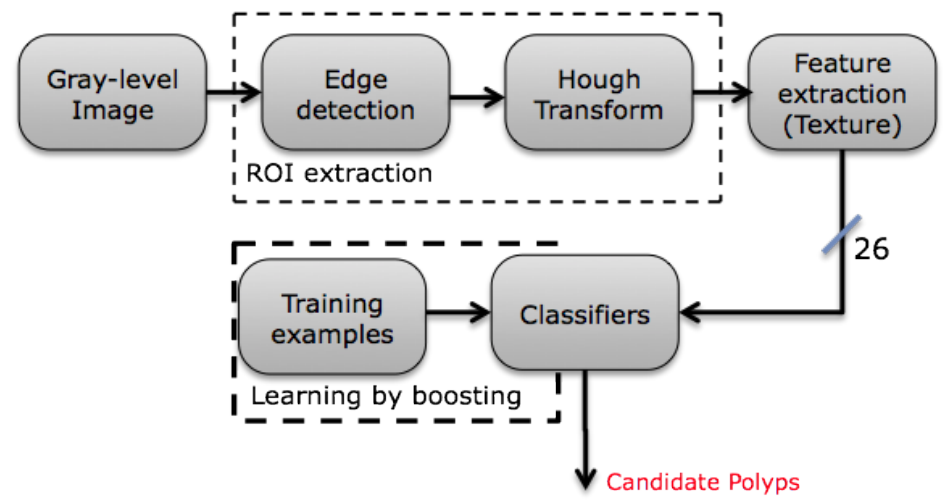

Fig. 4 Proposed diagram for the detection of polyps within videoendoscopy images. 
Geometric and Texture-based features

As mentioned before, the first useful characteristics for detection are size and shape of candidate structures. More precisely, a detection algorithm based on the circular form of the polyps is considered. Instead of considering a local curvature estimation or the Log-Gabor filtering, as suggested in [11], the circular Hough transform is used for three reasons; firstly, processing remains simple and efficient; secondly, all polyps must be detected even if numerous false positive ROI are also considered; thirdly, the Hough transform can be FPGA embedded like shown in [13] for an in situ and realtime detection. A discussion on that particular point is provided in the related section. In order to handle with different polyp sizes, we consider a research interval for the radii of the extracted circle.

For the texture-based analysis of pre-detected ROI, the co-occurrence matrix [14] is used to discriminate textural patterns of polyps and non-polyps. Main advantage of co-occurrence matrix is in their fixed dimensions only depending on the grey-scale resolution of images: as a consequence whatever is the dimensions of the candidate ROI, the size of the matrix remains the same, which is of first interest when considering the hardware implementation constraints (mainly memory) we have to deal with. Moreover, the textural discrimination capabilities of co-occurrence matrices remain of high efficiency even on grey-scale images [15] and could be implemented on FPGA [16] with possible limited memory resource, the 3 color channels being not necessary.

Basically, the cooccurence matrix $\mathcal{M C}_{\Delta x, \Delta y}(i, j)$ shows how often a pixel of greylevel value $i$ occurs either horizontally, vertically, or diagonally to adjacent pixels of value $j$ :

$$
\mathcal{M C}_{\Delta x, \Delta y}(i, j)=\sum_{p=1}^{n} \sum_{q=1}^{m}\left\{\begin{array}{l}
1, \text { if } I(p, q)=i \text { and } I(p+\Delta x, q+\Delta y)=j \\
0, \text { otherwise }
\end{array}\right.
$$

Twenty-six features (known as the Haralick's features [15]) are then extracted from each of the computed matrices. Are included : Contrast, Correlation, Entropy, Cluster Prominence, Cluster Shade, Dissimilarity, Homogeneity, Autocorrelation, Maximum probability, among other parameters (see Eqs. (2), (3), (4) for illustration of the first three parameters).

$$
\begin{aligned}
\text { Contrast } & =\frac{1}{K} \sum_{k=0}^{N-1} k^{2} \sum_{|i-j|=k} \mathcal{M C}(i, j), \\
\text { Correlation } & =\frac{1}{K \sigma_{x} \sigma_{y}} \sum_{i, j} i j M C(i, j)-\mu_{x} \mu_{y}, \\
\text { Entropy } & =-\frac{1}{K} \sum_{i, j} \mathcal{M C}(i, j) \log \left(\frac{\mathcal{M C}(i, j)}{K}\right),
\end{aligned}
$$

with $K$ the number of elements of $\mathcal{M C}(i, j)$ and 


$$
\begin{aligned}
\mu_{x} & =\frac{1}{K} \sum_{i, j} i * \mathcal{M C}(i, j) \\
\mu_{y} & =\frac{1}{K} \sum_{i, j} j * \mathcal{M C}(i, j) \\
\sigma_{x}^{2} & =\frac{1}{K} \sum_{i, j}\left(i-\mu_{x}\right)^{2} \mathcal{M C}(i, j) \\
\sigma_{y}^{2} & =\frac{1}{K} \sum_{i, j}\left(j-\mu_{y}\right)^{2} \mathcal{M C}(i, j)
\end{aligned} .
$$

Since the texture-based classification is performed using a boosting-based algorithm, no limitations about the number of parameters is considered, main idea being to let the learning process converge to the best classification solution without any prior information.

\section{Classification}

"Boosting" is a machine learning algorithm for supervised learning (see [17] among other publications of the same authors). It consists of the accumulation and constant learning of weak classifiers (a weak classifier is considered slightly correlated (a little better than chance) with the true classification), that once combined together generate a strong classifier, well-correlated with the ground truth provided by the expert. In the framework of our proposed approach, we use the boosting-based method of [18] setup in attentional cascade (Cascade Adaboost). This configuration allows us to create a strong classifier which performance can be priorly set-up in order to optimize the sensibility of the classification along with the specificity. For illustration of the overall learning algorithm, see figure 8 in which $F_{i}$ and $D_{i}$ stand for the maximum authorized False Positive Rate and the minimum acceptable detection rate, respectively, computed for each iteration of the process using the given $f$ and $d$ performance ratio, and $F_{\text {target }}$ the global false positive rate.

If the learning process related to boosting-based algorithms is time consuming, it is important to note that, once the optimal classifier is computed off-line, the classification step is very fast and fully compatible with a hardware implementation as shown by application to real-time face detection [18] embedded in cameras.

In our particular case, the considered weak classifiers are based on a set of truncated binary decision trees (bootstrapping) built from the 24 textural parameters on the dedicated learning database.

\section{Data}

Tests were performed on the database proposed by J. Bernal from the Universitat Autonoma de Barcelona [9], which consists of 300 videoendoscopy images presenting with one single polyp each, identified and segmented by a specialist. The data are courtesy made available by authors. To our knowledge, in the particular framework of 


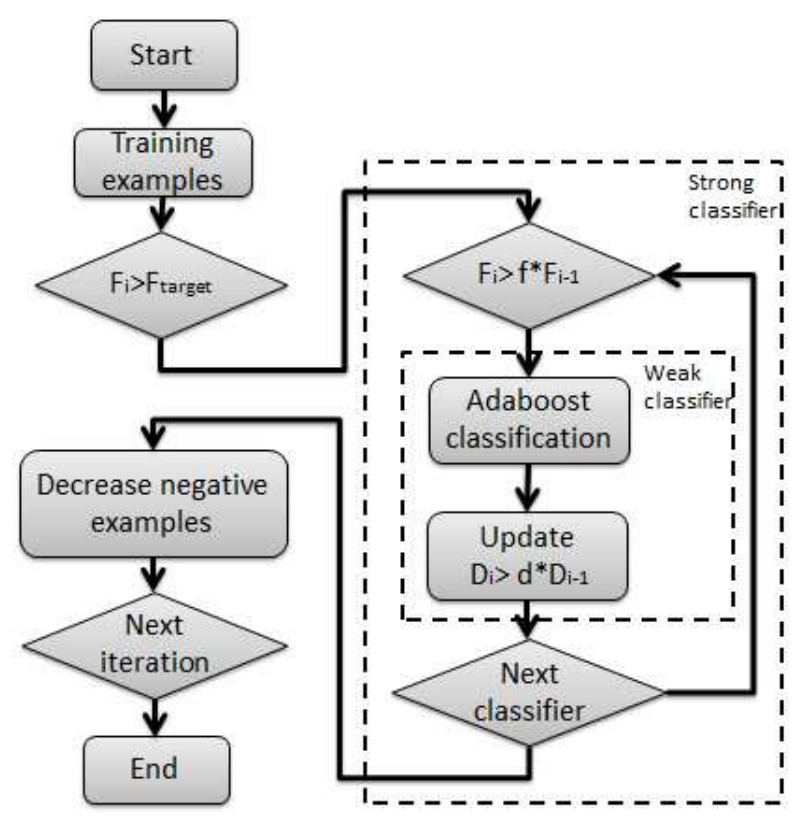

Fig. 5 Flow diagram that shows how the Cascade Adaboost is performed

colorectal polyp detections, this is currently the only existing on-line database with a sufficient amount of examples to be statistically meaningful. Figure 6 shows some example of polyps extracted from the database.

To build the learning database each image of the main dataset was sub-divided into five thumbnails by the gastroenterologist, as shown in figure 7. A first ROI corresponds to the polyp (a), and the other four to non-polyps (b-e). The resulting learning/testing database is then composed of a total of 1500 images, with 300 images of polyps and 1200 images of non-polyps, the labeling being performed,once again, by a specialist.

\section{Performance evaluation}

To proceed to performance evaluation of the proposed boosting-based method, three measures are usually considered meaningful and complementary: the sensitivity, the specificity and the false positive rate (FPR) respectively defined by:

$$
\begin{gathered}
\text { Sensitivity }=\frac{T P}{T P+F N}, \\
\text { Specificity }=\frac{T N}{T N+F P}, \\
F P R=\frac{F P}{F P+T N},
\end{gathered}
$$

with TP, FN, TN, FP standing for true positive, false negative, true negative and false positive. 

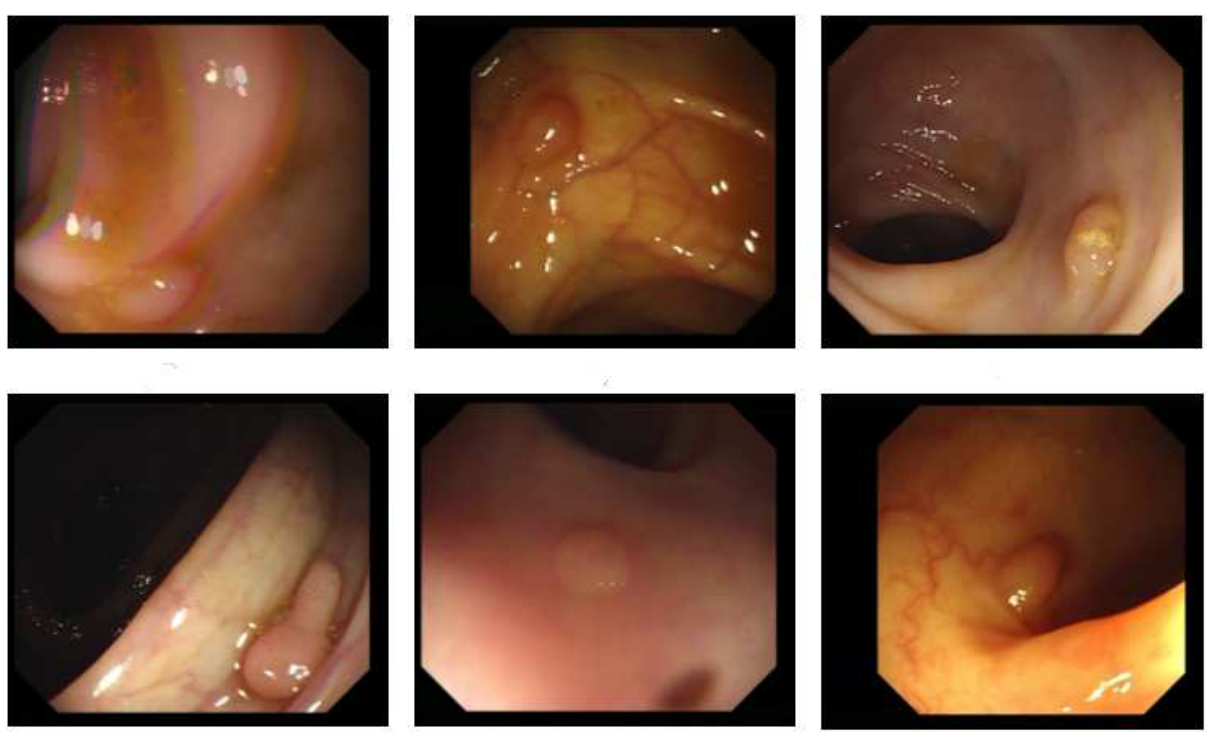

Fig. 6 Example of polyps extracted from the database of [9].

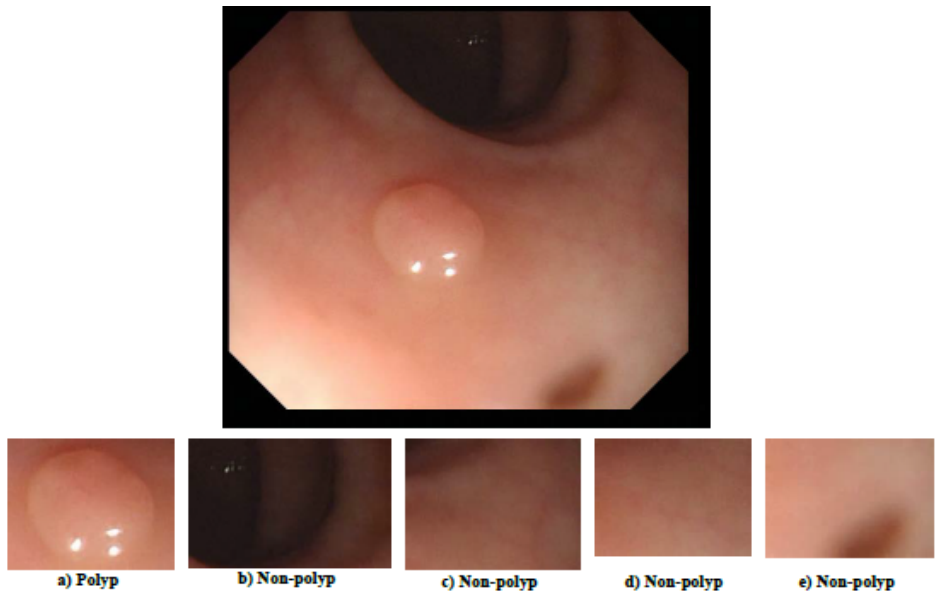

Fig. 7 Example on how the learning/testing database is generated from the original data of [9].

\section{Experiments}

Hough transform-based step

In table 2 the detection performance of the Hough transform on the aforementioned original database of [9] are shown and compared to the Log Gabor filtering proposed by $[11]$. 


\begin{tabular}{|l|c|r|}
\hline & Sensitivity & Specificity \\
\hline Hough transform & $94 \%$ & $15 \%$ \\
Log-Gabor & $42 \%$ & $89 \%$ \\
\hline
\end{tabular}

Table 2 Comparison of the detection sensitivity and specificity of the Hough transform and the Log Gabor filtering approach of [11] on the original database of Bernal et al.

We provide here the best obtained results considering the sensibility rate for an ad hoc set-up of the Hough transform circle detection threshold and for a research interval of the radii between 40 and 80 pixels.

We do not provide here usual Receiving Operating Curve (ROC) since we do not control the number of detected FP for a given threshold: Depending on the quality of the original image, number of FP can be very important (see figure 9.(c) for illustration).

At this stage, it can be noticed that the simple Hough transform allows a good detection of ROI containing a polyp even if the assumption made on the shape could be consider as restrictive since polyps are more elliptical than circular most of the times.

Moreover, if the value of specificity is low, the next classifying step will allow to improve the overall method performance.

\section{Learning-based classification performance using texture-based features.}

For these experiments, the ad hoc generated polyp/non-polyp database was divided into two subgroups: A first one composed of 1000 images (200 images of polyps and 800 of non-polyps) for the learning process and a second group for testing composed of the remaining 500 images. In order to obtain classification performance statistically meaningful, the drawing of the elements of both learning and testing databases were randomly made, and presented quantitative results correspond to the average value obtained on 100 different configurations.

In a first experiment, different kinds of methods for classification were compared: Learning Vector Quantization technic (LVQ) [19], classic Adaboost and finally Attentional Boosting (cascade adaboost). In terms of performance, as long as, contrary to cascade adaboost, it is not possible to set the obtained performance for LVQ or classic Adaboost, we privileged the balance between "Sensibility" and "Specificity". The results of this experimentation are shown in figure 8 .

As it can be noticed, among the different classification technics used, Cascade Adaboost provides the best compromise between "Sensibility" and "Specificity". If LVQ leads to a good classification of True Positive examples, the total amount of FPR remains too important considering the fact that $10 \%$ of the polyps are misclassified.

In a second experiment, only Cascade Adaboost is considered with a setting of the performance parameters $\left(F_{i}\right.$ and $D_{i}$ of figure 8$)$ chosen in order to have a "Sensibility" the closer to $100 \%$, whatever "Specificity" will be. This scenario fits better the expectations of radiologists who do not wish to miss possible polyps. Performance are shown in Table 3.

Tab. 3 shows that a high "Sensibility" is an objective that can be reached with a cascade adaboost setting of the learning process. Of course the "FPR" rate increases, 


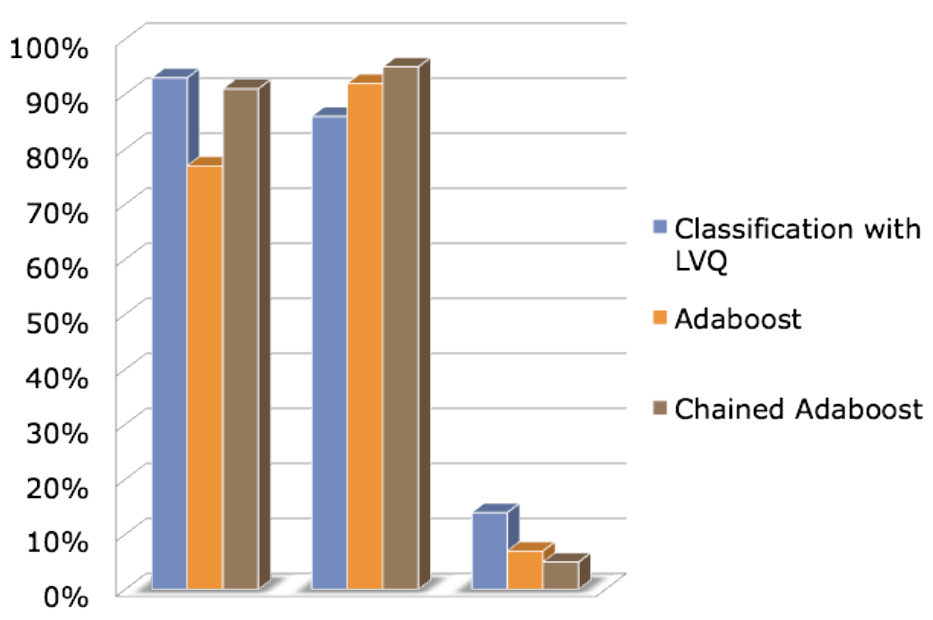

Fig. 8 Performance comparisons among different types of classification approaches, including adaboost and Cascade Adaboost. From left to right : Sensitivity, Specificity, False Positive Rate.

\begin{tabular}{|c|c|c|c|}
\hline Cascade Adaboost & Sensibility & Specificity & FPR \\
\hline \hline Mean & $99,5 \%$ & $86.1 \%$ & $13.9 \%$ \\
\hline Standard deviation & 0.00 & 0.07 & 0.07 \\
\hline
\end{tabular}

Table 3 Average performance of the Cascade Adaboost learning process with a "Sensibility" set to a minimum of $99 \%$.

but finally not that much considering the fact that for 100 polyps detected, only 14 more will be showed as possible candidates to the radiologist.

\section{Examples of detection and classification results}

In figure 9 some examples of detection/classification are shown. ROI that are skirted by a non-bolded plain rectangle are the ROI candidate issued from the Hough transform step of the proposed approach. ROI skirted by a bold plain rectangle are those which are effectively identified as a polyp after the texture-based classification.

In two first cases (a) and (b) of figure 9, the single polyp is detected and well classified. In the third image, where the polyp is even visually not that easy to detect due to the surroundings "noise", from nine ROI detected using the circular Hough transform, three are finally identified as polyp after the classification step, including the one containing the real polyp, generating two false positives.

\section{Simulation and time processing}

Up to now, all the developments have been performed under Matlab environment in order firstly to establish the feasibility of this new approach and to reduce the time development hardware. No optimization of the code has been realized. Currently, the 
time processing per image is about 2.05 seconds on a iCore 7 at $2.8 \mathrm{GHz}$. A multithreading version is under development both to accelerate by a factor 50 the time processing and to test first this approach in real time with the video output of a colonoscope before considering dedicated hardware implementation for WCE.

\section{Discussion}

Classification and Detection performance

A boosting based approach for polyp detection in videoendoscopy images has been proposed. Obtained results show interesting performance of the classification step in terms of selectivity and specificity, the most efficient learning method being the Cascade Adaboost one as shown in figure 8: As it can be noticed in figure 10, the boostingbased approach performed a classification which performance are close to Bernal et al's method. However, this latter comparison must be considered with cares since their proposed method and our are not exactly based on the same fundamental idea.

Moreover, detection results could be improved since currently the global detection rate of the overall processing scheme is only of $68 \%$ of the polyps: In figure 9.(c), it appears that when original image is corrupted by a strong acquisition noise, misclassifications can occur even if many FP ROI are discarded after the textural classification step. The remaining detected false polyps are errors probably made by the insufficient number of examples inside the database used for the learning step of the boosting method. Two main improvement will be considered in a close future: A first one consists in using a less sensitive detector than the circular Hough transform: the LoG (Laplacian of Gaussian) detector could be an alternative for instance even if the hardware implementation will need particular attention. A second point consists in improving the classification performance by integrating other features of interest (color, shape...), but also more training examples in the dataset to ensure a better representativity of the examples. This latter point could be achieved by proposing an off-line interactive process to the clinician that can add possible misclassified polyps to the learning database once the classification has been performed. Although such an approach could be timeconsuming because of the iteration of the learning process (boosting) each time a new example is added, such an effort should lead to an active extending of the available training database as well as an increase of the performance in terms of FPR [20].

Moreover, if presented results focus on classic videocolonoscopy, it appears necessary to build a training database dedicated to WCE images which characteristics (resolution, quality, etc.) can be quite different from the data used in this study. Nevertheless, we showed here that the learning-based approach can adapt to the particularity of used data by designing a proper learning database.

\section{Towards an integrated hardware implementation}

Currently, the Cyclope project only implements in hardware the SVM-based classification of 3D object with an FPGA Virtex II-pro [6]. Nevertheless, the algorithms proposed here can be also implemented on this platform considering the recent literature. Elhossini [21] proposed a memory efficient architecture for implementing Hough 
Transform on FPGAs. The proposed architecture enables storing the Hough Transform space on the FPGA's memory blocks with no need for accessing external memory while processing large size images in real-time with a 30 frame per second rate. Others hardware implementation allow to optimize the computation of angle by using Cordic algorithms, the time processing, the type (circle and/or line), etc. The main issue, is to develop a Hough Transform architecture that minimize the memory space with a great precision and a high parallelism. Table 4 summarizes the technical aspects of the five main contributions in the field.

\begin{tabular}{|c|c|c|c|c|c|}
\hline Authors & Year & Frame Size & Memory & Rate (FPS) & FPGA \\
\hline Jau Ruen [22] & 2006 & $256 \times 256$ & Ext. 1.6Mb & 0.2 & Altera Stratix 1 \\
\hline Souki $[23]$ & 2008 & $320 \times 240$ & Ext. 4Mb & 0.3 & Altera Cyclone 2 \\
\hline Geninatti $[24]$ & 2009 & $44 \times 46$ & Ext. 8Mb & 30 & Xilinx Spartan 3 \\
\hline Hardzeyeu [25] & 2008 & $500 \times 400$ & Ext. & 800 & Xilinx Virtex 2 \\
\hline Elhossini [25] & 2012 & $800 \times 600$ & Int. 250kb & 30 & Xilinx Virtex 2 \\
\hline
\end{tabular}

Table 4 Main characteristics of 5 of the most recent references of the literature.

Optimized embedded architecture based FPGA for an efficient and fast computation of grey level co-occurrence matrices (GLCM) and Haralick textures features for use in high throughput image analysis applications where time performance is critical have been already studied. The three main contributions [26] [16] [27] focus on the design of hardware processor that make possible to compute four distances $(1,2,3$ and 4 pixels) and four angles $\left(0^{\circ}, 45^{\circ}, 90^{\circ}\right.$ and $\left.135^{\circ}\right)$ in parallel. The main difference among these approaches lies in the strategy to address the neighboring pixel. Table 5 summarizes the performance of the three main recent contributions obtained on Virtex-II and Virtex-5 FPGA.

\begin{tabular}{|c|c|c|c|}
\hline Feature & Sieler [26] & Iakovidis [16] & Tahir [27] \\
\hline Year & 2010 & 2007 & 2004 \\
\hline FPGA & Virtex-5 & Virtex II & Virtex II \\
\hline ref & XC5VLX50T & XCV2000E & XCV2000E \\
\hline Frequency & $56.3 \mathrm{MHz}$ & $38.2 \mathrm{MHz}$ & $50 \mathrm{MHz}$ \\
\hline Area (FPGA used) & $21.9 \%$ & $45 \%$ & $59 \%$ \\
\hline Time processing (128x128) & $2.4 \mathrm{~ms}$ & $\mathrm{x}$ & $1.756 \mathrm{~ms}$ \\
\hline Ext. Mem & $\mathrm{x}$ & $800 \mathrm{~kb}$ & $327 \mathrm{~kb}$ \\
\hline Int. Mem & $327 \mathrm{~kb}$ & $83.2 \mathrm{~kb}$ & $81.9 \mathrm{~kb}$ \\
\hline
\end{tabular}

Table 5 Main characteristics of related work.

Boosting classification has been also implemented in hardware on FPGA [28]. According to the related work on the hardware implementation of Hough Transform, co-occurence matrices computation and boosting classification, it is feasible to embed our approach on FPGA circuit.

This first step is a pre-required one towards an ASIC design embedded in the WCE, but also to be able to precisely estimate the energy consumption related to the hardware implemented detection/classification algorithms. In a previous work, we demonstrated that $75 \%$ of the power consumption of a smart RF sensor are due to 
the RF power budget [29]. Moreover, [30] showed the transmitter power consumption is a non linear function of the data rate and concluded that to increase the battery life of a smart sensor, the amount of data should be reduced. The overall gain of an intelligent transmission versus a continuous transmission in a standard WCE (see figure 1) depends on the one hand on the estimation of the number of images (polyps and false positives) that will be transmitted and on the other hand, on the power consumption due to their processing.

Currently, we can make only an estimation of this overall gain based on hypothesis, because of the integrated circuit has not been yet designed and the in vivo experiments not achieved. We are working on the hardware implementation of the $2 \mathrm{D}$ classification on a FPGA-based platform. By considering the above state of the art, the fact that an FPGA also consumes 12 times more dynamic power than an equivalent ASIC on average [31] and the power consumption of Virtex 5, we can estimate that the power consumption due to the processing will be approximatively under the hundred of $\mu W$. This feature is less than the power consumption of the usual eight white LED used for illumination and the RF transceiver.

Moreover, during a standard examination, around 50000 images are sent to the data logger with a frame rate of $4 \mathrm{fps}$ up to $35 \mathrm{fps}$. By considering the same examination with the possible presence of ten polyps and a FPR of $13.9 \%$, only 6960 images will be sent and a 7 factor can been won on the overall power consumption of the transmission RF.

\section{Conclusion}

In this paper, we introduced a new embeddable method for polyp detection in videoendoscopic examinations. The entire detection chain combines geometric and textural features for polyp characterization: if the first geometric step remains simple with the use of the Hough transform, the textural features computed from co-occurrence matrices are integrated within a boosting-based approach making possible to achieve good classification performance similar to those of the most recent state-of-the-art article. At last, the complete developed detection/classification scheme is in accordance with a hardware implementation which is of primary importance for possible in situ application using WCE.

\section{Conflict of interest}

Juan S. Silva, Aymeric Histace, Olivier Romain, Xavier Dray and Bertrand Granado declare that they have no conflict of interest.

\section{References}

1. M. C. Parkin F.J. Shin, B.F. Forman, "Globocan 2008 v1.2, cancer incidence and mortality worldwide: Iarc cancerbase no. 10.," International Agency for Research on Cancer, 2008.

2. A. Moglia, , A. Menciassi, A. Dario, and A. Cuschieri, "Capsule endoscopy: progress update and challenges ahead," Nature reviews. Gastroenterology 65 hepatology, , no. 6, pp. 352-362, June 2009. 
3. Cristiano Spada, Cesare Hassan, Miguel Munoz-Navas, Horst Neuhaus, Jacques Deviere, Paul Fockens, Emmanuel Coron, Gerard Gay, Ervin Toth, Maria Elena Riccioni, Cristina Carretero, Jean P. Charton, Andr Van Gossum, Carolien A. Wientjes, Sylvie SacherHuvelin, Michel Delvaux, Artur Nemeth, Lucio Petruzziello, Cesar Prieto de Frias, Rupert Mayershofer, Leila Aminejab, Evelien Dekker, Jean-Paul Galmiche, Muriel Frederic, Gabriele Wurm Johansson, Paola Cesaro, and Guido Costamagna, "Second-generation colon capsule endoscopy compared with colonoscopy," Gastrointestinal Endoscopy, vol. 74 , no. 3, pp. $581-589,2011$.

4. A. Bergwerk, D. Fleischer, and J. Gerber, "A capsule endoscopy guide for the practising clinician: technology and troubleshooting," Medline, vol. 66, no. 6, pp. 1188-1195, Dec. 2007.

5. R. et al. Eliakim, "Prospective multi center performance evaluation of the second generation colon capsule compared with colonoscopy," Endoscopy, vol. 41, pp. 1026-1031, 2009.

6. A. Kolar, O. Romain, J. Ayoub, S. Viateur, and B. Granado, "Prototype of Video Endoscopic Capsule With 3-D Imaging Capabilities," Biomedical Circuits and Systems, IEEE Transactions on, vol. 4, no. 4, pp. $239-249,2010$.

7. J. Ayoub, B. Granado, Y. Mhanna, and O. Romain, "SVM based colon polyps classifier in a wireless active stereo endoscope," in 2010 IEEE EMBC, 2010, pp. 5585 -5588.

8. M. Liu, L. Lu, J. Bi, V. Raykar, M. Wolf, and M. Salganicoff, "Robust large scale pronesupine polyp matching using local features: A metric learning approach," in Medical Image Computing and Computer-Assisted Intervention MICCAI 2011, Gabor Fichtinger, Anne Martel, and Terry Peters, Eds. 2011, vol. 6893 of Lecture Notes in Computer Science, pp. 75-82, Springer Berlin Heidelberg.

9. J. Bernal, J. Sanchez, and F. Vilariño, "Towards automatic polyp detection with a polyp appearance model," Pattern Recognition, vol. 45, no. 9, pp. 3166 - 3182, 2012.

10. P. N. Figueiredo, I. N. Figueiredo, S. Prasath, and R. Tsai, "Automatic polyp detection in pillcam colon 2 capsule images and videos: Preliminary feasibility report," Diagnostic and Therapeutic Endoscopy, , no. 182435, pp. 7 pages, 2011.

11. A. Karargyris and N. Bourbakis, "Identification of polyps in wireless capsule endoscopy videos using log gabor filters," in IEEE Workshop LiSSA, april 2009, pp. 143 -147.

12. V. Kodogiannis and M. Boulougoura, "An adaptive neurofuzzy approach for the diagnosis in wireless capsule endoscopy imaging," Int. J. of Information Technology, vol. 13, pp. 46 $-56,2007$.

13. S. Tagzout, K. Achour, and O. Djekoune, "Hough transform algorithm for fpga implementation," Signal Processing, vol. 81, no. 6, pp. 1295 - 1301, 2001.

14. Larry S. Davis, Steven A. Johns, and J. K. Aggarwal, "Texture analysis using generalized co-occurrence matrices," Pattern Analysis and Machine Intelligence, IEEE Transactions on, vol. PAMI-1, no. 3, pp. $251-259$, july 1979.

15. R.M. Haralick, "Statistical and structural approaches to texture," Proceedings of the IEEE, vol. 67, no. 5, pp. 786-804, 1979.

16. D. K. Iakovidis, D. E. Maroulis, and D. G. Bariamis, "Fpga architecture for fast parallel computation of co-occurrence matrices," Microprocess. Microsyst., vol. 31, no. 2, pp. 160-165, Mar. 2007.

17. R.E. Schapire and Y. Singer, "Improved boosting algorithms using confidence-rated predictions," Mach. Learn., vol. 37, no. 3, pp. 297-336, Dec. 1999.

18. S. Viola and M. Jones, "Rapid object detection using a boosted cascade of simple features," in Proceedings of the 2001 IEEE CVPR Conference, 2001, pp. 511-518.

19. T. Kohonen, The Handbook of Brain Theory and Neural Networks, chapter Learning vector quantization, MIT Press, Cambridge, MA, 1995.

20. M. Liu, L. Lu, X. Ye, S. Yu, and M. Salganicoff, "Sparse classification for computer aided diagnosis using learned dictionaries," in Medical Image Computing and Computer-Assisted Intervention MICCAI 2011, Gabor Fichtinger, Anne Martel, and Terry Peters, Eds. 2011, vol. 6893 of Lecture Notes in Computer Science, pp. 41-48, Springer Berlin Heidelberg.

21. A. Elhossini and M. Moussa, "Memory efficient fpga implementation of Hough transform for line and circle detection," in CCECE, 2012, pp. 1-5.

22. J. J. Ruen, M. S. Shie, and C. Chen, "A circular hough transform hardware for industrial circle detection applications," in Industrial Electronics and Applications, 20061 ST IEEE Conference on, 2006, pp. 1-6.

23. M.A. Souki, L. Boussaid, and M. Abid, "An embedded system for real-time traffic sign recognizing," in Proceedings - 2008 3rd International Design and Test Workshop, IDT 2008, pp. $273-276$. 
24. S.R. Geninatti, S.R. Benavidez-Benitez, M. Hernandez-Calvino, N. Guil-Mata, and J. Gomez-Luna, "Fpga implementation of the generalized hough transform," in Proceedings - 2009, International Conference ReConFigurable Computing and FPGAs, pp. $172-177$.

25. V. Hardzeyeu and F. Klefenz, "On using the hough transform for driving assistance applications," in 2008 International Conference on Intelligent Computer Communication and Processing, 2008, pp. $91-98$.

26. L. Sieler, C. Tanougast, and A. Bouridane, "A scalable and embedded FPGA architecture for efficient computation of grey level co-occurrence matrices and haralick textures features," Microproc. and Microsys., vol. 34, no. 1, pp. $14-24,2010$.

27. M.A. Tahir, A. Bouridane, and F. Kurugollu, An FPGA Based Coprocessor for the Classification of Tissue Patterns in Prostatic Cancer, vol. 3203 of Lecture Notes in Computer Science, pp. 771-780, Springer Berlin Heidelberg, 2004.

28. J. Mitéran, J. Matas, E. Bourennane, M. Paindavoine, and J. Dubois, "Automatic hardware implementation tool for a discrete adaboost-based decision algorithm," EURASIP Journal on Applied Signal Processing, vol. 2005, pp. 1035-1046, 2005.

29. A. Suissa, O. Romain, J. Denoulet, K. Hachicha, and P. Garda, "Empirical method based on neural networks for analog power modeling," Trans. Comp.-Aided Des. Integ. Cir. Sys., vol. 29, no. 5, pp. 839-844, May 2010.

30. A.Y. Wang and C.G. Sodini, "On the energy efficiency of wireless transceivers," in Communications, 2006. ICC '06. IEEE International Conference on, 2006, vol. 8, pp. 3783-3788.

31. I. Kuon and J. Rose, "Measuring the gap between fpgas and asics," Trans. Comp.-Aided Des. Integ. Cir. Sys., vol. 26, no. 2, pp. 203-215, Feb. 2007. 

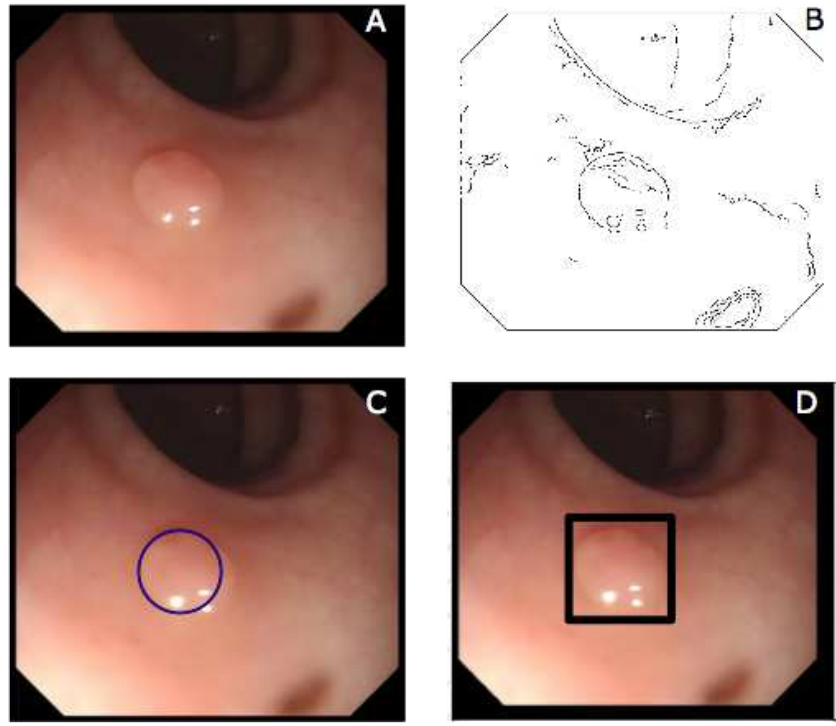

(a)
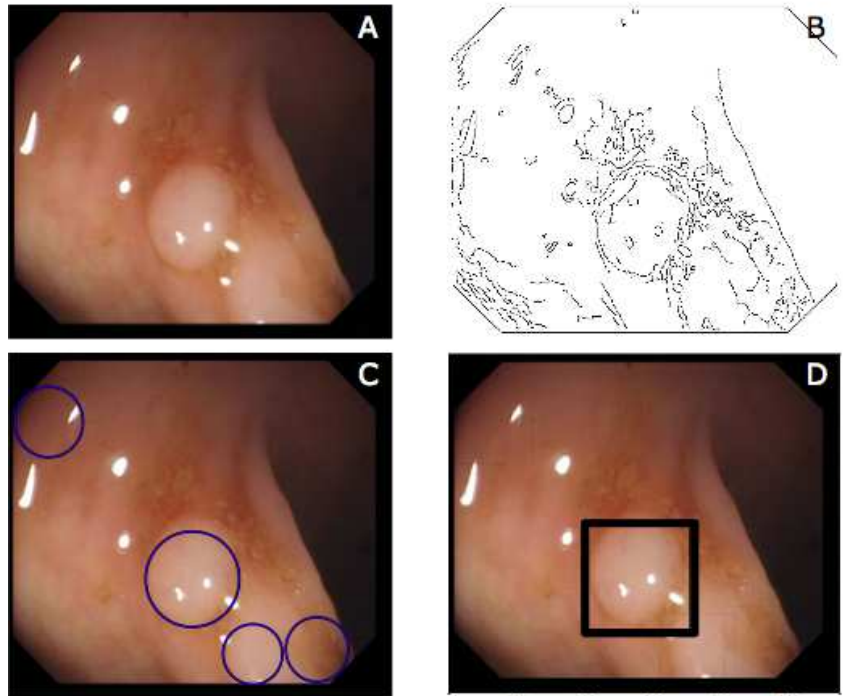

(b)
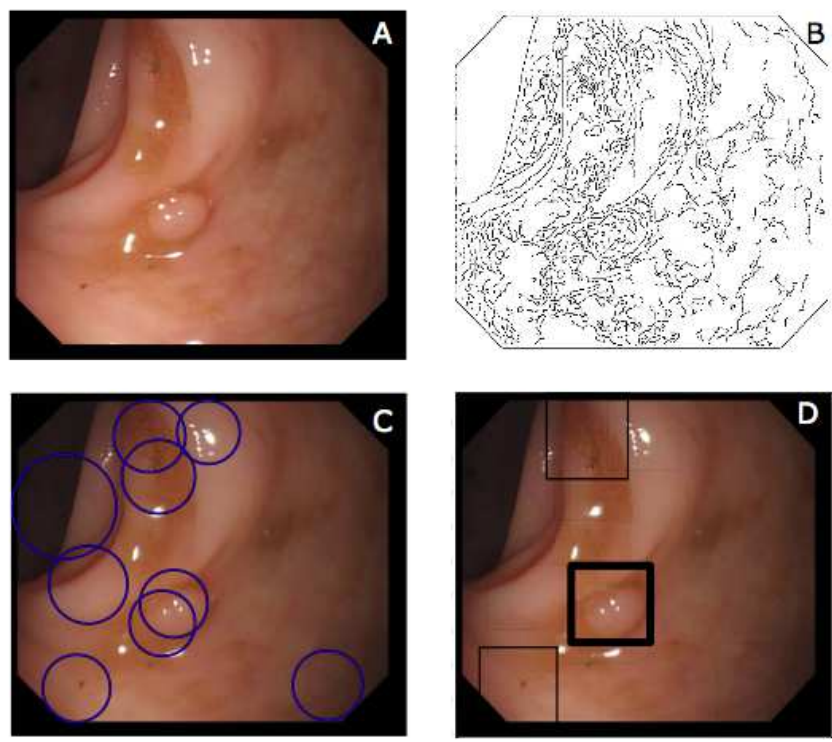

(c)

Fig. 9 Three examples of detection/classification of polyps in three different images extracted from the original database of [9]. For all images: A, Original image ; B: Boundary detection using a Canny-Deriche filter ; C, ROI obtained using the circular Hough transform detector ; $\mathrm{D}$, ROI classified as a polyp using the learning based proposed approach. 


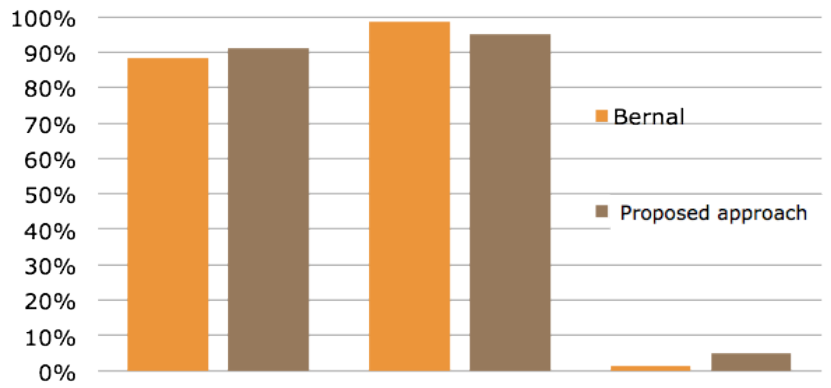

Fig. 10 Comparison between the method exposed by Bernal [9] and the attentional boosting based classification method proposed: from left to right: Sensitivity, Specificity and FPR 